FILOZOFIA

Roč. 76, 2021, č. 6

DOI: https://doi.org/10.31577/filozofia.2021.76.6.2

\title{
TOWARDS A UNIVERSAL ACCOUNT OF ASYMMETRY IN NON-CAUSAL EXPLANATIONS
}

LUKÁŠ ZÁMEČNÍK, Univerzita Palackého v Olomouci, Filozofická fakulta, Katedra obecné lingvistiky, Olomouc, $\check{C} R$

\begin{abstract}
ZÁMEČNÍK, L: Towards a Universal Account of Asymmetry in Non-causal Explanations

FILOZOFIA, 76, 2021, No 6, pp. $407-422$

In this paper, I provide a conceptual analysis of the issue of asymmetry in noncausal explanations. I aim to fulfill three sub-tasks: 1) To define a conceptual space for problem solving (Cf. Reutlinger and Saatsi 2018; Lange 2017); 2) To reflect ongoing debates (Cf. Jansson and Saatsi 2019; Lange 2019; Khalifa et al. 2018); 3) To reflect a model of topological explanation (Cf. Kostić 2020). I focus only on non-causal explanations in the context of scientific research. I formulate several counter-arguments against distinctively mathematical explanations. I reflect the ongoing debate on the question of the universal applicability of a counterfactual solution to the problem of asymmetry for both causal and non-causal explanations (Cf. Woodward 2018). I reveal that none of the approaches discussed is without problems and that the proposed solutions to them are unsatisfactory. I consider the model of topological explanation in Kostić's view to be the most cogent solution to the discussed problem. His model successfully incorporates a counterfactual approach and also offers a solution to the problem of explanation asymmetry in non-causal explanations from a pragmatic point of view.
\end{abstract}

Keywords: Asymmetry - Explanation - Non-causal - Counterfactual - Topological

\section{Motivation}

Debates in recent years have been focused on the topic of non-causal explanations, and have now reached the stage of evaluation and recapitulation (Reutlinger and Saatsi 2018; Lange 2017) after a period of collecting examples from individual sciences. In addition to a more precise classification of non-causal explanations, ${ }^{1}$ a clearer definition of the pragmatic aspects of the use of non-causal explanations (Bokulich 2018), and the reopening of the debate on the nature of the causal nexus (Woodward 2018), they have also raised some fundamental questions that return to general characteristics

\footnotetext{
${ }^{1}$ There are non-causal explanations in particular sciences (physics, life sciences, chemistry, etc.), non-causal explanations in the form of distinctively mathematical explanations of non-mathematical facts, non-causal explanations in the form of explanations in mathematics, and last but not least metaphysical explanations (Lange 2017).
} 
of scientific explanations. One of these characteristics is the asymmetry of scientific explanation - more precisely the asymmetric relation between the explanans and explanandum, where the entities in the explanans explain the entities in the explanandum, but not vice versa.

The basic distinguishing ingredient ensuring that the relationship between explanans and explanandum remains asymmetric has been recognized in causality. The flagpole, represented by its height and volume in general, is the cause of the existence of a shadow due to the nature of the light rays, while the opposite cannot be said. In general, following Khalifa et al. (2018) from the 1960s (in connection with Bromberger 1966 and other criticisms by Salmon 1998), the focus shifted from the emphasis on inference (present in the DN model of explanation) to trying to capture causal nexus between entities described in explanans and entities described in the explanandum. ${ }^{2}$ In the canonical form, we find the causal conception of scientific explanation in Woodward's (2003) counterfactual framework.

The asymmetry problem is important for new debates, mainly because it may indicate that the inability of some non-causal explanations to ensure an asymmetric relation of explanans and explanandum is a sign of the invalidity of these explanations. What's more, it can completely undermine the validity of distinctively mathematical explanations. Moreover, this problem can also be an obstacle for non-causal explanations in general.

Woodward (2018) offers a conservative solution to the asymmetry problem, based on a counterfactual approach, and Jansson and Saatsi (2019) build on this. They believe that it is possible to modify the counterfactual expression of the asymmetry of explanation in causal cases for non-causal cases. Lange (2019) puts forward serious arguments against this conservative solution but does not offer a clear solution to the problem.

In this paper, I set out three tasks. The first task (in Chapter 2) is to define a conceptual space wherein it makes sense to ask a question of explanatory asymmetry. I will try to show some specific problems of a distinctively mathematical explanation. The second task (in Chapter 3) is to document in more details this ongoing key debate expressed in Jansson and Saatsi (2019) and Lange (2019). The common problem of both these approaches is that they consider the question of the asymmetry of explanation somewhat in isolation from the specific scientific explanations themselves on somewhat artificial examples (i.e. bridges in Königsberg), which are linked to the distinctive role of mathematics in scientific explanation. Therefore, the third task (in Chapter 4) is a reflection on Kostić's (2020) concept of topological explanation, which

\footnotetext{
${ }^{2}$ Halvorson (2016) associates with this the "prejudice" that in the syntactic view of theories, as well as in the models of explanation typical for the received view of the philosophy of science, the entities in the explanans and explanandum are viewed as linguistic entities.
} 
removes the shortcomings of the approaches of Jansson and Saatsi (2019) and Lange (2019) mentioned above.

\section{Narrowing the framework of the problem}

Given all the shades of reasoning over explanations, ${ }^{3}$ the concept of non-causal explanation is also very broad. Because the current philosophy of science does not reject questioning of the relation between metaphysics and logic, logic and mathematics, and also mathematics and particular sciences (and of course all possible other relations between these conceptual systems), the issue of non-causal explanations is raised in many different contexts. One of the positives of this pluralistic approach is that the concept of explanation has been much more finely defined than it was in the traditional, received view. However, my goal is not to examine the problem of the asymmetry of explanations in this full range, but in the more traditional context of the empirical sciences. For this reason, some frequently asked questions remain aside - it is primarily a concept of mathematical and metaphysical explanation. ${ }^{4}$

The specific case of a non-causal explanation is a distinctively mathematical explanation (DME). It is important in two respects: on the one hand, it is referred to by participants in debates on the asymmetry of explanation (Jansson and Saattsi 2019; and Lange 2019), and on the other hand, some explanations present in physics belong to it (in particular, explanations based on space-time symmetries and generally on gauge symmetries). An example of DME has become the Strawberry Problem (SP): The purely mathematical fact (23 is an odd number) seems to explain a physical fact (a mother cannot divide 23 strawberries between three children without slicing) (see Lange 2017,6). Asymmetry in this case could be connected to the higher modal force of the mathematical fact (Lange 2019, 23 - 24).

The problem with the Bridges of Königsberg is, probably, the most popular example of DME. Lange asks a question:

Why has no one ever succeeded (or, in particular, why did a given person on a given occasion not succeed [we have to add that it is valid for the specific arrangement from the year 1735, a note of the author]) in crossing all of the bridges of Königsberg exactly once (while remaining always on land or on a bridge rather than in a boat, for instance, and while crossing any bridge completely once having begun to cross it)?

And Lange gives a graph-theoretical DME of this fact:

\footnotetext{
${ }^{3}$ The current philosophy of science is characterized by a wide degree of the plurality of concepts of explanation, but little ability to unify this plurality into general "theories of explanation".

${ }^{4}$ E.g. Poggiolesi forthcoming, Lange 2017, XIV-XX.
} 
A distinctively mathematical explanation is that in the bridge arrangement considered as a network, it is not the case that either every vertex or every vertex but two is touched by an even number of edges (Lange $2017,7-8)$.

Many arguments have been raised against DME (i.e. Morrison 2015, 50 - 57; Erik Weber ${ }^{5}$ 2019). In addition, I will add two other argument sketches. Firstly, it seems that distinctively mathematical explanations, at least in the case of SP, are ad hoc explanations. It is not possible to inter-connect them in an explanatory system, as we are used to, for example, from the elaborate theories of physics. ${ }^{6}$ I believe that a necessary condition for a valid explanation is that every explanation must be included in the hierarchy of scientific explanations, or must indicate the place in the hierarchy of explanations that the explanation occupies. The question is whether this condition can be fulfilled in the case of distinctively mathematical explanations.

Secondly, what if I reverse the perspective? Isn't it more the case that physical objects in the case of SP demonstrate the property of "being an odd number"? I think this is analogous to, for example, demonstrating the validity of the Pythagorean theorem. We can be considering this the case of school homework. Children will receive two equal-sized paper squares, on which they draw lines with the help of a ruler, as shown in the diagram:

\section{Scheme 1}
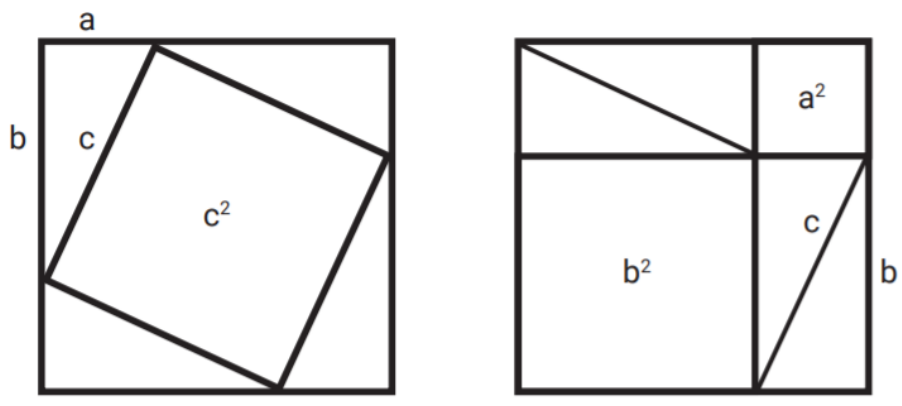

Giere $(2006,10)$

\footnotetext{
${ }^{5}$ In the lecture "Against 'Distinctively Mathematical' Explanations of Physical Facts" given at the workshop „Non-Causal Explanations: Logical, Linguistic and Philosophical Perspectives“ in Gent, May $10^{\text {th }}, 2019$.

${ }^{6}$ A strategy that would, for example, link all explanations through odd numbers to the general property of "oddity" and then possibly link them to some axiomatization of natural numbers, does not seem adequate to me.
} 
Some children become conscious of the idea behind the Pythagorean theorem due to the visualization, but it is better to let children cut both squares according to the drawn lines. By comparing the resulting shapes, they simply find that the four triangles from the left square are the same size as the four triangles from the right square. This means, of course, that what was left after removing these triangles from the left square - the square above the hypotenuse - is as large as what was left after removing these triangles from the right square - the squares above both legs. The children can then add all three squares above the respective sides to one more triangle and then repeat the statement of the Pythagorean theorem.

Ronald Giere (2006) argues that in this way we come to understand mathematical claims; that we grasp sophisticated abstract models of mathematics through similar schematizations, diagrams, and visualizations. These considerations are part of Giere's conception of distributed cognitive systems. It is essential for us that Giere uses directly the formulation that these diagrams: "[...] are used to prove the Pythagorean Theorem" (Giere 2006, 101). Here, Giere completes his pragmatic conception of theories in a peculiar way by its relation to cognitive science - all our scientific models are somehow based on our cognitive activity. Understanding mathematics is also governed by cognitive models - the basis of understanding mathematics is not deduction and proof, but the use of a visual aid that illustrates an abstract statement.

In Lange's (2017) conception of distinctively mathematical explanation, on the other hand, it is a mathematical fact, structure, or statement that in some cases explains non-mathematical facts (in physics, biology, or linguistics). In the case of the Pythagorean theorem and children's experiment that leads to its understanding, we can argue that a mathematical statement - the Pythagorean theorem - explains why a child who gets two different piles of cut paper can make an equal square of each.

In other words, in this case, the Pythagorean theorem does not come into consideration at all. A child just manages to assemble two squares of the same size. An observer familiar with mathematics (and distinctively mathematical explanations) can say that the Pythagorean theorem explains the success of this childlike endeavor. Just as a generalized statement about the Euclidean plane can explain that the author of an ancient Roman mosaic managed to create a mosaic of just $n$ stone pieces. Would we not then be in a situation where any configuration of physical objects can be explained distinctively mathematically by some mathematical fact? I think so, and I think that this indicates the vagueness of the concept of a distinctively mathematical explanation.

\section{The debates about the asymmetry in non-causal explanations}

I will now focus on summarizing the arguments about the possibilities of expressing asymmetry in the non-causal explanations presented in the current discussions by Jansson and Saattsi (2019), Lange (2019). At the same time, it will be clarified why I dealt with distinctively mathematical explanations above. 
Jansson and Saatsi (2019) focus on the 'explanatory abstractions', which occur precisely in non-causal explanations, but not only in them. The authors insist that "all" explanations somehow include abstractions (to varying degrees) (Jansson and Saatsi 2019,822 ) and can be expressed by modifying Woodward's Why-questions (and counterfactual dependencies) (Jansson and Saatsi 2019, 824). Their core idea is that we can understand the dependence of the explanandum on the explanans (but not vice versa) as a unifying condition for all causal and non-causal explanations. I think this idea implies that asymmetry is an expression of the explanatory power of explanation.

The authors distinguish three variants of abstractions, and these variants express: 1) independence from the actual structure of entities; 2) independence from actual laws; and 3) the degree of generalizability of the statement in the explanans (for example, from 23 strawberries to strawberries in a quantity of prime numbers, etc.) (Jansson and Saatsi 2019, 821 - 822). The difference between causal and abstract explanations is not given absolutely but by the degree of presence of abstractions of type $1-3$ (Jansson and Saatsi 2019, 823).

The core of the argument is that abstractions in the sense of 1 and 2 are not explanatory because they do not provide information about dependence (between the explanans and explanandum) while the third type of abstraction can be linked to Woodward's concept of explanation based on counterfactual dependence (Jansson and Saatsi 2019, 824). The authors identify "change-relating counterfactuals" (CRC) - a subset of counterfactuals and at the same time a broader class than the interventionist causal counterfactuals that Woodward works with. They are convinced that CRC expresses an asymmetry indicating the dependence of the explanandum on the explanans, but not vice versa (Jansson and Saatsi 2019, 835) - the point that Lange's (2019) arguments attack (see below).

Jansson and Saatsi state:

In the counterfactual framework, explanations must involve an invariant, change-relating generalization that supports counterfactuals indicating an explanatory dependence of the explanandum on the explanans (Jansson and Saatsi 2019, 837).

According to the authors, the well-known example of the non-causal explanation, "Königsberg's Bridges", can be understood as an abstraction of the third type (Jansson and Saatsi 2019, 841). That is, it is possible to choose an intuitive explanation: "there must be an even number of bridges", but this intuitive explanation is limited, although still explanatory via the change-relating counterfactual, unlike Euler's fully graphtheoretical explanation which includes all cases, for example, for any number of vertices and edges of the graph.

It can be seen that these conclusions coincide with my above statement about the ad hoc nature of distinctively mathematical explanations. We will see that it is this 
feature of DME that Lange (2019) tries to dampen in order to preserve the originality of the explanatory nature of mathematical abstractions. It seems to me that for Jansson and Saatsi $(2019,841)$ the abstract explanations sui generis (i.e. explanation based on abstractions only) simply do not exist. At the same time, they believe that a unifying Woodward counterfactual framework can be used for all types of explanations (Jansson and Saatsi 2019, 842).

This is an attractive way of solving the problem: we can defend the asymmetry of explanation in Woodward's framework because there are no strictly non-causal explanations. Woodward's approach can be applied because there is still the possibility to intervene to the (non-abstract) conditions in explanans, quite similarly like in Weber's view (see above). Here, of course, I generalize beyond the expressions about the distinctively mathematical explanations. However, I will try to find a less radical solution to the problem of asymmetry (following Kostić 2020).

In general, Lange (2019) - unlike Jansson and Saatsi (2019) - does not trust the universality of the counterfactual approach to explanatory asymmetry. Moreover, he does not believe that it is possible to find another uniform solution to the problem of explanatory asymmetry:

[...] there is no fully general account of what makes some facts explanatorily prior to others in non-causal scientific explanations. Rather, the order of explanatory priority is fixed by different considerations in different noncausal explanations (Lange 2019, 24).

According to Lange, the explanatory priority can be given through: 1) greater modal strength (for example, meta-laws over first-order laws); 2) metaphysical choice (for example, the substantivalist conception of spacetime can put explanatory priority on the number of dimensions of space over the stability of the solar system), 3) the belief that facts about reality have explanatory priority over facts about appearances (Lange 2019, 24). A certain disadvantage of Lange's text is that Lange rejects particular solutions to the problem of asymmetry, based on a counterfactual framework, but he leaves his proposals of explanatory priority without further justification.

The main argument against Woodward's view (Woodward 2018) is based by Lange on the example used by Jansson and Saattsi (2019), "Königsberg's Bridges". The point is that Woodward chooses an example where $\mathrm{N}$ (non-traversability) is modal in nature and cannot be the subject of intervention, unlike $\mathrm{E}$ (number of vertices). In such a case, we can use (as Lange agrees) a counterfactual approach, but this would significantly narrow the set of non-causal explanations used in science (Lange 2019,5 - 6). Lange points to cases where both the explanans and explanandum are 
modal in nature (Lange 2019, 7) and where asymmetry is lost because neither can be intervened (he offers an example from node theory, Lange 2019, 7 - 8).

Lange $(2019,10)$ disagrees with the proposal by Jansson and Saatsi (2019) because he finds a counterexample to their variant of counterfactual expression of asymmetry. Respectively, Lange argues that the success of their strategy depends on the choice of variables, to show that:

[...] J\&S's approach yields the correct verdict regarding the strawberry example only because of what seems like a rather incidental feature of that example: that although the explanans would have been different, had the explanandum been different, there is no specific way that the explanans would have been different (Lange 2019, 10).

Lange concludes, with reference to Jansson (2015), that counterfactual asymmetry often accompanies explanatory asymmetry (that both asymmetries exist in parallel), but that it does not mean that counterfactual asymmetry is a source of explanatory asymmetry (Lange 2019, 11).

Some of Lange's arguments seem artificial, ${ }^{7}$ but his general argument tries to capture the core of the problem by showing that the counterfactual approach works well in causal cases of explanation, because they always have asymmetries fixed. In this regard, Lange speaks of the asymmetry of the "causal arrow" and illustrates it with an example:

Decisions by bridge designers and builders $\rightarrow \sim \mathrm{E} \rightarrow \mathrm{N}$ (Lange 2019, 15).

That is to say, decisions of bridge builders in Königsberg $\rightarrow$ bridges in Königsberg do not have Eulerian properties $(\sim$ E) $\rightarrow$ bridges in Königsberg do not have traversability $(\mathrm{N})$.

Lange points out that the first arrow is causal (and is indeed an arrow), but the second arrow (expressing a non-causal explanation) is not causal and can be understood as: 1) a logical necessity, but then the relation between $\sim \mathrm{E}$ and $\mathrm{N}$ becomes symmetric; and 2) a counterfactual dependence, but it is between $\sim \mathrm{E}$ and $\mathrm{N}$ (despite Woodward 2018) also symmetric (Lange 2019, 15). Lange adds:

If the second arrow is understood simply as non-causal explanation, then it is asymmetric $(\rightarrow$, not $\longleftrightarrow)$. But in an account of non-causal explanation,

\footnotetext{
${ }^{7} \mathrm{He}$ admits this when he criticizes the article by Jansson and Saattsi (2019): "I do not want to put much weight on this" tricky "change of variables" (Lange 2019, 10).
} 
such an arrow cannot be presupposed; it must be accounted for (Lange 2019, 15).

Lange clearly reveals the fundamental difficulty associated with the counterfactual approach to asymmetry, according to his judgment. In the case of causal explanations, the counterfactual approach to asymmetry works well because the causal nexus itself (causal arrow) takes care of this asymmetry. However, nothing like this is possible in non-causal cases, where we could postulate asymmetry (to say that there is a non-causal arrow), but that would reverse our task (see the previous quote).

In the second part of the text, Lange (2019) deals with the explanation of not singular facts but of laws (and regularites they entail, Lange 2019, 16). However, Lange makes his counter-arguments de facto by referring to scientist consensus, or preference for certain explanatory strategies:

[...] scientific practice of explaining regularities is often very uniform in embracing certain directions of explanation rather than others. Famously, for example, spacetime symmetry principles are regarded as explaining conservation laws rather than the reverse (Lange 2019, 19).

This shows the traditional "conflict" of prescriptive and descriptive approaches to the tasks of philosophy of science. Jansson and Saatsi (2019) seek a universal interpretation of asymmetry through counterfactual means. They are willing to "sacrifice" specific habits which, in the case of scientific practice, favor certain asymmetrical relations between the explanans and explanandum, and others lag behind. Lange (2019), on the other hand, describes the scientific practice and shows individual examples in their diversity rather than the universal concept of asymmetry.

This pluralism is then expressed by Lange in the final part of the text, which notes the explanatory asymmetry in the case of the relation between spacetime symmetries and conservation laws in physics, with references to French and Saatsi (2018). Lange claims that spacetime symmetries have an explanatory priority in physics because they are conceived as meta-laws on which the principles of conservation are based, but not vice versa (Lange 2019, 23).

Here I disagree with Lange because we can reflect on the practice of the scientific community and notice the preferences that arise in favor of some explanatory strategies (see below Kostić's view), but this is not the same as treating the philosophical generalizations of some physicists (Brian Greene or Eugene Wigner, see Lange 2019, 23 ), as if it were a consensus present in the scientific community. I do not think that there is any practically proven conceptualization of meta-laws among physicists. The 
same applies to the other two ways of justifying the explanatory asymmetry that Lange presents (see above 2) and 3)).

On the contrary, I believe that a certain consensus (expressed in textbooks, see Stenger 2006) would be based on the cosmological principle. Here the spacetime symmetries (and generally gauge symmetries) play a fundamental role. Because the validity of symmetries of a certain type ensures the point-of-view invariance of the laws of physics and so ensures the comprehensibility of the world for any observer in a uniform way. However, again, even for this consensual conception of the explanatory priority of spacetime symmetries, it is true that no matter how obvious this consensus is in physics, it is a classical approach. ${ }^{8}$ And it is true that many interesting things symmetry breakings, emergent phenomena, universality, etc. - in contemporary physics often take place where this classical approach occurs at the limit of its possibilities. ${ }^{9}$

In summary, in my opinion, it can be argued that Lange does not solve the problem of asymmetry in non-causal cases in general because he does not offer a universal pattern for asymmetric non-causal explanations (Lange would agree), but also does not offer convincing arguments even for partial cases. Only for the first procedure (the modal strength), we can conclude there is a kind of common agreement about the hierarchy of physical laws (like "the momentum invariance" is higher in the hierarchy than "Newton's third law"). However, even in this case, we can hardly claim that there is a common concept of meta-law in physics. ${ }^{10}$ Procedures 2 and 3 , on the other hand, step out of the scientific framework which interests me - metaphysical choice, as well as differentiating statements about reality and appearances, are not something that would play an explicit role in theory-building in the scientific community. These are not explicit parts of scientific theories that serve to explain. ${ }^{11}$

Therefore, it seems to me that the counterfactual approach is "purer", paving a path that is universal, prescriptive in the traditional sense of received view of the philosophy of science and does not deviate from scientific discourse. Its disadvantage is that it changes our intuition about what is explanatory, what has explanatory priority. However, that should be expected from the philosophy of science. That its conceptual analysis contributes to the transformation of some of our ideas, intuitions and myths. In Lange's arguments, there is an emphasis on those parts of the philosophy of science that are so autonomous on the actual operation of science that their decision-making

\footnotetext{
${ }^{8}$ For example, how does the time asymmetry expressed by the Big Bang theory fit into this? I thank Jiř́ Langer who once asked me this question.

${ }^{9}$ This is a problem of choosing the appropriate type of symmetry and a problem of symmetry breaking. Morrison (2013) writes about this in connection with considerations about three types of unification in physics: reductive and synthetic unity (we can conceive as classical approach) and universality. ${ }^{10}$ May be in some meta-theoretical ideas of physicists, like Krauss $(2012,174-175)$ who claims that the laws of quantum mechanics should be valid in the whole inflating multiverse.

${ }^{11}$ Here I could enter into discussions about the role of metaphysics in physics (see Maudlin 2010), but this is not the aim of the study.
} 
role vis-à-vis scientific praxis is - I am afraid - illusory. Next, however, I will focus on whether the counterfactual approach to asymmetry is sufficient.

\section{Kostić's conception of topological explanation}

By analysis of Jansson and Saatsi (2019) and Lange (2019), I mapped the situation in the current debates on explanatory asymmetry in non-causal explanations. Jansson and Saatsi (2019) are convinced of the universal validity of the counterfactual expression of explanatory asymmetry, while Lange (2019) does not believe in a universal solution to the problem of explanatory asymmetry and bases the partial solutions on a meta-theoretical level. ${ }^{12}$

I believe that Kostić (2020) is closest to solving the problem. His approach is specific in that he does not look for a universal variant of asymmetry for all explanations, but consistently conceptualizes the model of topological explanation. He builds it in a counterfactual form, ${ }^{13}$ defines for it a clear form of asymmetry (in several variants, see below) and shows its applicability in the biological sciences. This is the way a philosopher of science works which is in concordance with my belief that conceptual analysis should be useful for scientific practice. ${ }^{14}$

Kostić constructs in the counterfactual framework the general theory of topological explanations, which looks like this:

$A$ topologically explains $B$ if and only if:

1) (Facticity): $A$ and $B$ are approximately true; and

2) Either

a) (Vertical mode): $A$ describes a global topology of the network, $B$ describes some general physical property, and had $A$ had not obtained, then $B$ would not have obtained either; or

b) (Horizontal mode): $A$ describes a set of local topological properties, $B$ describes a set of local physical properties, and had the values of $A$ been different, then the values of $B$ would have been different.

3) (Explanatory perspectivism): $A$ is an answer to the relevant explanation-seeking question $\mathrm{Q}$ about $B$, such that the $\mathrm{Q}$ determines whether to use vertical or horizontal explanatory mode (Kostić $2020,2) .^{15}$

\footnotetext{
${ }^{12}$ Meta-laws, metaphysical choices, etc. (Lange 2019, 24).

${ }^{13}$ This overcomes the difficulties of Huneman's model of topological explanation (Cf, Huneman 2018, 2010).

${ }^{14}$ This is a matter of "philosophical preferences", however, it grows up from the pragmatic view of the philosophy of science (e.g. Cartwright 1999, Giere 2006).

${ }^{15}$ As an example we can choose Kostić's topological explanation of cognitive brain control: "When explaining cognitive control in either mode, the brain is represented as a network of brain regions connected through white matter tracts. In the vertical mode, the explanans is the global topological
} 
In connection with this model of explanation, Kostic proposes three variants of explanatory asymmetry: property, counterfactual, and perspectival (Kostić 2020, 5), while he comments only briefly on the first two. The most important type of asymmetry for Kostic is perspectival asymmetry, and this is also, in my opinion, the most important benefit of Kostić's concept of topological explanation. It is based directly on the third point of Kostić's theory of topological explanation: "A is an answer to the relevant explanation-seeking question Q about B" (Kostić 2020, 2), to which we can add, "[...] but not a vice versa."

Kostić demonstrates perspectival asymmetry for both vertical and horizontal cases of counterfactual dependence on examples from current researches in neuroscience. ${ }^{16} \mathrm{He}$ thus presents examples of how counterfactual dependence and explanationseeking questions establish an asymmetry of topological explanations (Kostić 2020, 6-7). The scientist is able to defend his explanation-seeking strategy in a form that satisfies the counterfactual dependence (more precisely, a philosopher of science is able to construct this counterfactual "above" the "considerations" of a scientist who seeks to explain the phenomenon/class of phenomena).

Kostić's main strategy is revealed when he invokes the concept of "scientific interest":

[...] explanation-seeking questions are relevant, in part, because they serve scientists' interests. Often, the properties that interest scientists have asymmetries that are highly specific to the phenomena they are studying (Kostić 2020, 5).

A pragmatic conception of theories is evident in Kostić's conception of perspective asymmetry (Cf. Giere 2004). It does not matter that someone explains spacetime symmetry by the laws of conservation and someone is explaining the laws of conservation by spacetime symmetries, it is important that the asymmetry of explanation arises in every particular example of scientific explanation in which the explanation-

property such as small-worldliness and the explanandum is the global physical property such as global cognitive control (whether global cognitive control is theoretically possible). [...] Smallworldliness as a global topological property indicates that almost any two nodes in the network will be connected either directly or through a minimal number of indirect connections, which shortens the distance between the nodes within a neighbourhood of nodes as well as between neighbourhoods of nodes, and neighbourhoods of neighbourhoods, which further ensures that the energy requirements for changing any of the trajectories will be minimal, and thus explains why the network is globally or in principle controllable" (Kostić 2019, 4).

${ }^{16}$ These are two current examples from neuroscience: "[...] asymmetry of information flow (Seguin et al 2019), or asymmetry of topological organization of the auditory cortex (Mišić et al 2018)" (Kostić 2020, 11). 
seeking question is well expressed. Asymmetry is then an element explicitly introduced into the scheme of explanation only through a specific scientific interest. I interpret Kostić's preference for perspective asymmetry as an inclination to this pragmatic conception of explanation.

Unlike the previous authors, Kostić's ambitions are much more sober. He tries to show the universal applicability of topological explanation in biology, respectively in scientific strategies that work with models of biological networks. Such a procedure is conceptually appealing - he tries to use a minimum of meta-theoretical means. It is also appealing for practical reasons - he reflects on a whole large area of current biological research (see examples: Kostić 2020, 6-7), for which he provides a methodological framework. Explanatory asymmetry acquires practical significance, orients scientists to clarify their research priorities and research questions.

I am afraid that wanting more presupposes "subscribing" to some of the paths suggested by Lange (see variants $1-3$ above). I, therefore, conclude with (provisional) reflections on the asymmetry of explanation by claiming that I consider Kostić's explanatory perspectivism to be satisfactory from a practical point of view in accordance with the pragmatic view of theories. It represents the path chosen by scientists to find answers to their explanation-seeking questions. ${ }^{17}$

\section{Conclusions}

I focused on the problem of asymmetry of explanations, which acquired its specific form in their non-causal variant. In the search for a solution to this problem, I decided to exclude some notions of non-causal explanations - metaphysical explanations and mathematical explanations in mathematics - in order to remove the excessive complexity of the conceptual field. I have also expressed some reservations about some variants of the distinctively mathematical explanation, although, in general, I believe it cannot be omitted when solving the problem of asymmetry.

On a purely conceptual level, I did not find a clear and universal solution to the mentioned problem. By gradually reflecting on the most important current attempts at a solution (Janssson, Saatsi 2019; Lange 2019; and Kostić 2020), I "only" revealed the basic pitfalls that prevent finding a general solution. Jansson and Saatsi's (2019) effort to create a universal counterfactual variant of solving the asymmetry problem runs into Lange's (2019) argument. Unfortunately, Lange himself (2019) does not try to find a universal solution, moreover, he does not specify his variants of the solution $(1-3)$.

\footnotetext{
17 There is indeed an affinity of Kostić's and Lange's view of non-causal explanations - these approaches do not offer a universal solution for all variants of explanation. However, unlike Lange, Kostić conceives a universal model of topological explanation where the explanatory asymmetry stems from the pragmatic maxim - the scientist is the source of explanation-seeking questions.
} 
In general, it seems that counterexamples can be found for all solution cases (and Lange excels in this regard). The basic difficulty is actually seen in the traditional form of the problem, which was demonstrated by the flagpole problem: it is a conflict of inference versus dependence (for example, causality). We can see this in the different approaches of Khalifa et al. (2018) versus Lange (2019). The former is looking for an inference basis for the explanatory framework - and somehow additionally looking for solutions to asymmetry - while Lange is looking for new frameworks for expressing a dependency that will exhibit an asymmetric relation. De facto, it is roughly the same problem that we find in Hempel's inference concept in the D-N model (versus causality, also known as Salmon). The purely inference model (Khalifa et al. 2018) argues with the one that needs metaphysical help (Lange 2019).

We find a solution based on counterfactual dependence in Kostić (2020) for the case of a topological explanation. A closer look, and taking into account Kostić's assertion that he could not remove Lange's objections (Kostić 2019, 4), we find that the strongest element of Kostić's model is perspectival asymmetry, for which counterfactual asymmetry represents "only" interpretative means because this is where it would be possible to apply Lange's argument against the sufficiency of counterfactuals. Perspectival asymmetry based on the choice of explanation-seeking question, which reveals the interests of the author of the explanation, a particular scientist, leads us to a pragmatic notion of explanation, based on a pragmatic conception of scientific theory. I, therefore, argue that explanatory asymmetry is established by a scientist through his/her explanation-seeking question, he/she establishes the direction of the explanation by choosing the support on which he/she builds his theory or model. ${ }^{18}$

In this text, I have tried to propose a perspective solution, further based on a pragmatic conception of theories (and models). Of course, this will not satisfy those who, together with Lange (also Jansson and Saatsi), strive for a clean conceptual solution. The idea that it is the researcher's own intention, which prioritizes some parts of the explanatory structure and imprints the desired asymmetry on it, is not an evasive maneuver. The way of solving the problem of explanatory asymmetry will probably always be influenced by the way we conceive the theory as we primarily see it: whether as an inference structure (syntactic view), a system of models representing data (semantic view), or by means chosen by the researcher to solve the explanation-seeking question (pragmatic view).

This perspective solution will not convince these philosophers who believe that we can grasp the metaphysical facts about reality itself. I have to state that my empirical stance (Fraassen 2002) is completely different - I do not believe we can grasp

${ }^{18}$ I completely agree with an anonymous reviewer that another interesting problem tightly connected with the pragmatic point of view is: "[...] how scientist can design his explanation with purpose to persuasively communicate it in scientific community and to incorporate it in a wider theoretical framework (which does not only rely on his sequential, contextual and temporary chosen W-question)". 
the metaphysical realm - I believe that we are bound in our "transcendental limits". If a solution of explanatory asymmetry should be found on a syntactic-semantic basis of the view of scientific theories, ${ }^{19}$ then it would be necessary to conceive a principlebased view of explanation, in which the principle guarantees the explanatory power of the explanans. And these principles - identified in particular sciences - are connected with the point-of-view invariance principle (the cosmological principle, see above). This view of explanation will also use counterfactual "scaffolding", will prioritize inference and will work specifically with causal and non-causal cases of explanations.

\section{Bibliography}

BOKULICH, A. (2018): Searching for Non-Causal Explanations in a Sea of Causes. In: Reutlinger, A. -Saatsi, J. (eds.): Explanation Beyond Causation: Philosophical perspectives on noncausal explanations. Oxford: Oxford University Press, $(141-163)$.

BROMBERGER, S. (1966): Why-questions. In: Colodny, R. (ed.): Mind and Cosmos. University of Pittsburgh Press, $(86-111)$.

CARTWRIGHT, N. (1999): The Dappled World: A Study of the Boundaries of Science. Cambridge: Cambridge University Press.

FRAASSEN, B. C. (2002): The Empirical Stance. London: Yale University Press.

FRENCH, S., SAATSI, J. (2018): Symmetries and explanatory dependencies in physics. In: Reutlinger, A. - Saatsi, J. (eds.): Explanation Beyond Causation: Philosophical perspectives on non-causal explanations. Oxford: Oxford University Press, (185-205).

GIERE, R. N. (2006): Scientific Perspectivism. Chicago: The University of Chicago Press.

GIERE, R. N. (2004): How Models Are Used to Represent Reality. Philosophy of Science, 71 (5), $742-752$.

HALVORSON, H. (2016): Scientific Theories. In: Humphreys, P. (ed.): The Oxford Hanbook of Philosophy of Science. Oxford: Oxford University Press, (pp. $585-608$ ).

HEMPEL, C. G. (1965): The Logic of Functional Analysis. In: Hempel, C. G.: Aspects of Scientific Explanation and other Essays in the Philosophy of Science. New York: The Free Press, (297330).

HUNEMAN, P. (2018): Diversifying the picture of explanations in biological sciences: ways of combining topology with mechanisms. Synthese 195 (1), $115-146$.

HUNEMAN, P. (2010): Topological explanations and robustness in biological sciences. Synthese, $177(2), 213-245$.

JANSSON, L. \& SAATSI, J. (2019): Explanatory Abstractions. Brit. J. Phil. Sci, 70 (3), 817 - 844

KHALIFA, K., MILLSON, J., RISJORD, M. (2021): Inference, Explanation, and Asymmetry. Synthese 198, 929 - 953. DOI: https://doi.org/10.1007/s11229-018-1791-y

KOSTIĆ, D. (2020): General Theory of Topological Explanations and Explanatory Asymmetry. Philosophical Transactions B of the Royal Society. DOI: https://doi.org/10.1098/ rstb.2019.0321

KOSTIĆ, D. (2019): Mathematical and non-causal explanations: an introduction. Perspectives on Science, 27 (1) 1 - 6.

KRAUSS, L. M. (2012): A Universe from Nothing. New York: Free Press.

LANGE, M. (2019): Asymmetry as a challenge to counterfactual accounts of non-causal explanation. Synthese 198 (4). DOI: https://doi.org/0.1007/s11229-019-02317-3

\footnotetext{
${ }^{19}$ Halvorson (2016) writes in an engaging and visionary way about the interconnectedness of these approaches.
} 
LANGE, M. (2017): Because Without Cause: Non-Causal Explanations in Science and Mathematics. Oxford: Oxford University Press.

MAUDLIN, T. (2010): The Metaphysics within Physics. Oxford: Oxford University Press.

MORRISON, M. (2015): Reconstructing Reality: Models, Mathematics, and Simulations. Oxford: Oxford University Press.

MORRISON, M. (2013): The Unification in Physics. In: Batterman, R. (ed.): The Oxford Handbook of the Philosophy of Physics. Oxford: Oxford University Press, (381 - 415).

POGGIOLESI, F. (2020): Grounding principles for relevant implication. Synthese DOI: https:// doi.org/10.1007/s11229-019-02523-z

REUTLINGER, A., SAATSI, J. (2018): Explanation beyond causation: Philosophical perspectives on non-causal explanations. Oxford: Oxford University Press.

SALMON, W. (1998): Causality and Explanation. Oxford: Oxford University Press.

STENGER, V. (2006): The Comprehensible Cosmos: Where Do the Laws of Physics Come From? New York: Prometheus Books.

WOODWARD, J. (2003): Making Things Happen: A Theory of Causal Explanation. Oxford University Press.

WOODWARD, J. (2018): Some varieties of non-causal explanation. In: Reutlinger, A. - Saatsi, J. (eds.): Explanation Beyond Causation: Philosophical perspectives on non-causal explanations. Oxford: Oxford University Press, (117 - 140).

GAČR (2019-2021): Simplifying Assumptions and Non-Causal Explanations, number GA19$04236 \mathrm{~S}$.

Lukáš Zámečník

Univerzita Palackého v Olomouci

Filozofická fakulta, Katedra obecné lingvistiky

Křížkovského 14

77900 Olomouc

Česká republika

e-mail: lukas.zamecnik@upol.cz

ORCID ID: https://orcid.org/0000-0002-0965-4583 